\title{
Synthesis models for starburst populations with Wolf-Rayet stars
}

\author{
Claus Leitherer \\ Space Telescope Science Institute ${ }^{1}$, \\ 3700 San Martin Drive, Baltimore, MD 21218, USA
}

\begin{abstract}
The prospects of utilizing Wolf-Rayet populations in starburst galaxies to infer the stellar content are reviewed. I discuss which WR star features can be detected in an integrated stellar population. Specific examples are given where the presence of WR stars can help understand galaxy properties independent of the O-type star population. I demonstrate how populations with small age spread, such as super star clusters, permit observational tests to distinguish between single-star and binary models to produce WR stars. Different synthesis models for WR populations are compared. Predictions for WR properties vary dramatically between individual models. The current state of the models is such that a comparison with starburst populations is more useful for improving WR atmosphere and evolution models than for deriving the star-formation history and the initial mass function.
\end{abstract}

\section{Wolf-Rayet signatures in young populations}

The central 30 Doradus region has the highest concentration of Wolf-Rayet stars in the LMC. Parker et al. (1995) classify 15 stars within $20^{\prime \prime}$ (or $5 \mathrm{pc}$ ) of R 136 as WR stars, including objects which may appear WR-like due to very dense winds (de Koter et al. 1997). This suggests that about 1 out of 10 ionizing stars around R 136 is of WR type. The WR stars can be seen in an ultraviolet (UV) drift-scan spectrum of the integrated 30 Dor population obtained by Vacca et al. (1995). Their spectrum is reproduced in Fig. 1. The He II $\lambda 1640$ emission is from the WR population, demonstrating that WR signatures can be seen superimposed on the integrated spectrum of the OB population.

While Fig. 1 suggests that WR features should be detectable, it is also clear that they will not be very prominent in comparison with O-star features. WRand O-stars occupy roughly the same part of the Hertzsprung-Russell diagram, therefore they have similar $L$ and $T_{\text {eff }}$, whereas WRs have lifetimes of only $10 \%$ those of $\mathrm{O}$ stars, and they tend to descend from more massive (less frequent) $\mathrm{O}$ stars. Taken together, this implies that any spectral tracer of WR stars that is present in O stars as well will not be useful to identify WR stars in an unresolved population.

Three key properties distinguish WR from O stars: (i) WR mass-loss rates $(\dot{M})$ are one to two orders of magnitude higher so that wind-sensitive lines are strongly in emission. Equally important, the EUV spectrum above 4 Ryd is

\footnotetext{
${ }^{1}$ Operated by AURA, Inc., for NASA under contract NAS5-26555
} 


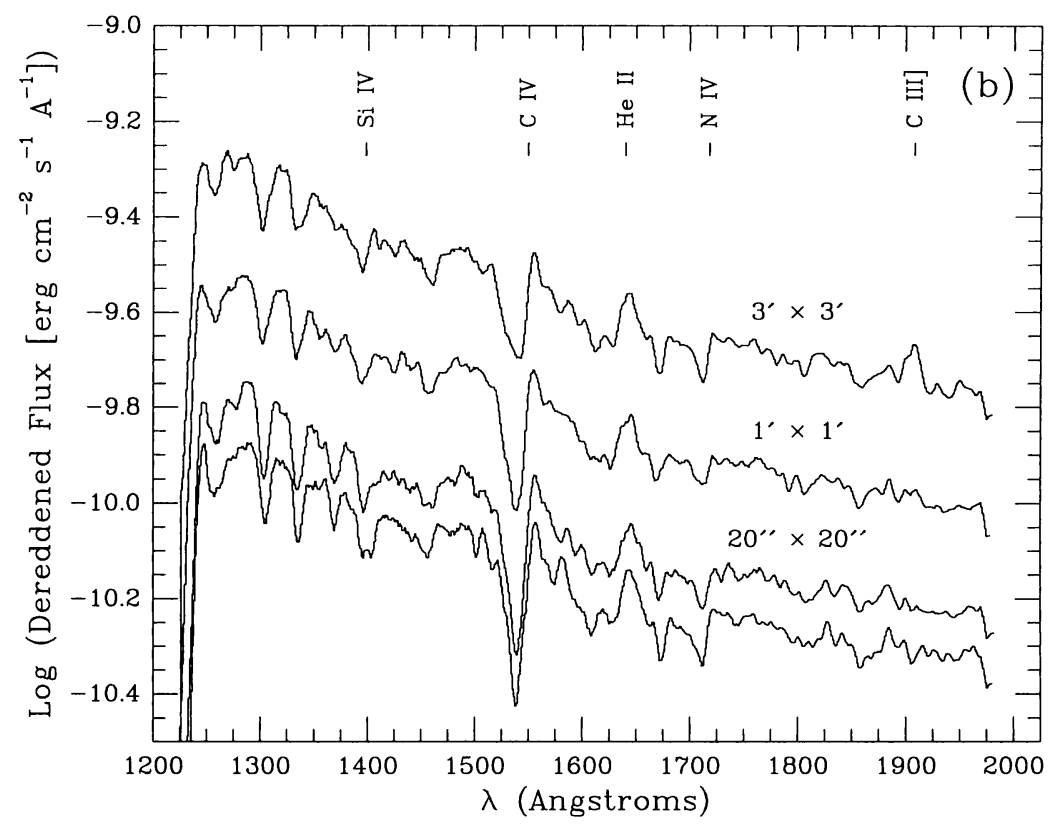

Figure 1. IUE drift-scan spectrum of the central 30 Doradus region (Vacca et al. 1995).

highly enhanced due to wind-induced recombination (Gabler et al. 1989) so that WR stars are an important source of energetic photons; (ii) WR stars are more chemically evolved. Therefore spectral lines sensitive to nuclear processing, like those of $\mathrm{He}, \mathrm{C}, \mathrm{N}$, and $\mathrm{O}$, become prominent in WR stars; and (iii) WR stars are formed with a time delay after $\mathrm{O}$ stars are observed. The time delay depends on the details of the gas stripping from the surface, either radiatively/pulsationally by stellar winds (Conti 1976) or by gravitation in interacting binaries (Vanbeveren et al. 1998).

Table 1. WR contribution in a 5 Myr old population $\left(Z=\mathrm{Z}_{\odot}\right)$.

\begin{tabular}{|c|c|c|c|c|c|c|c|}
\hline Parameter & $\begin{array}{c}W(\mathrm{H} \alpha) \\
(\AA)\end{array}$ & $\begin{array}{c}W(4686) \\
(\AA)\end{array}$ & $\begin{array}{c}F_{\lambda}^{1500} \\
\quad(\mathrm{erg}\end{array}$ & $\begin{array}{c}F_{\lambda}^{5550} \\
-1 \mathrm{~cm}^{-2}\end{array}$ & $\begin{array}{l}F_{\lambda}^{22000} \\
\left.\AA^{-1}\right)\end{array}$ & $\begin{array}{c}\dot{M}_{\text {winds }} \\
\left(\mathrm{M}_{\odot} \mathrm{yr}^{-1}\right)\end{array}$ & $\begin{array}{c}L_{\text {winds }} \\
\left(\mathrm{erg} \mathrm{s}^{-1}\right)\end{array}$ \\
\hline from WR & $45 \%$ & $100 \%$ & $5 \%$ & $12 \%$ & $22 \%$ & $97 \%$ & $98 \%$ \\
\hline
\end{tabular}

These properties are the basis of WR synthesis models. Such models predict how wind-sensitive WR lines, like stellar and nebular He II $\lambda 4686$ or stellar C IV $\lambda 5808$ vary with age and other fundamental properties of the population (e.g., Schaerer \& Vacca 1998). Table 1 illustrates this point. This table lists the contribution from WR stars to various observables of a $5 \mathrm{Myr}$ old single stellar population of solar composition. The predictions are from the STARBURST99 package (Leitherer et al. 1999). I chose $t=5 \mathrm{Myr}$ since the WR contribution 
reaches a maximum at this epoch. WR stars are important via their winds, both thermally $(\lambda 4686)$ and non-thermally $\left(\dot{M}\right.$ and mechanical luminosity $\left.L_{\text {winds }}\right)$, have some minor contribution to the $\mathrm{H}$-ionizing photon flux, and are negligible for the UV-optical-IR continuum. (The quoted numbers are conservative upper limits since I assumed that the 'WR-free' comparison population has zero contribution from WR stars. A more realistic approximation would be replacing WR stars by $\mathrm{O}$ stars.) The observational strategy, therefore, is to search for, e.g., broad and narrow $\lambda 4686$ or for $\lambda 5808$ in starburst populations to probe the WR content (e.g., Schaerer et al. 1999)

\section{Wolf-Rayet stars - are they useful?}

One may ask what we can learn from the detection of WR stars in a starburst population that could not be inferred from the O-star population as well. After all, counting ionizing photons from $\mathrm{O}$ stars via recombination radiation requires much less observational effort than the detection of a faint, broad WR feature. There are, however, notable cases where the detection of WR stars has played a pivotal role for the interpretation of the stellar content of a galaxy.

$W R$ stars in active galactic nuclei. Hydrogen recombination radiation is most commonly taken as an indicator of massive stars. There are instances where nebular properties are difficult to relate to the hot-star content. Theoretical and observational evidence has been accumulating over the past years that starbursts play an important role in the Seyfert galaxy phenomenon (Perry \& Dyson 1985; Terlevich \& Melnick 1985; Cid Fernandes \& Terlevich 1995). The UV-to-near-IR continuum in Seyfert 2 nuclei has been suggested to originate from stars, as opposed to being reflected light from the hidden Seyfert 1 nucleus. OBA stars, the suspected continuum source have few, if any, suitable lines which would not coincide with strong nebular emission lines (an exception being the UV; see González-Delgado et al. 1998). On the other hand, the emission lines are not predominantly due to stellar photoionization, making them unsuitable as a hot-star indicator. Therefore the discovery of WR features in Mrk 477 (Heckman et al. 1997), Mrk 1210 (Storchi-Bergmann et al. 1998), and other Seyfert 2 galaxies (D. Kunth, these Proceedings) is significant: simple population synthesis analysis using the WR content suggests that stars produce the featureless continuum.

The porous and dusty interstellar medium. Even in the absence of an AGN, nebular properties are sometimes difficult to interpret in terms of the ionizing population. 'Photon counting' is complicated by photon leakage and absorption of photons by dust. The latter effect may be important in IR-luminous galaxies whose ionizing radiation field is known to be particularly soft (Goldader et al. 1997; R. Joseph, these Proceedings). NGC 1614 falls into this category. Its observed radiation field can only be understood if the typical mass of the ionizing star is in the $20-30 \mathrm{M}_{\odot}$ range (Doyon et al. 1992; Lançon \& Rocca-Volmerange 1996), which translates into a stellar initial mass function (IMF) truncated at $\sim 30 \mathrm{M}_{\odot}$. Yet NGC 1614 has been reported as a WR galaxy by Conti (1991). Unless stellar evolution proceeds differently in this galaxy, the analogy with other WR galaxies suggests a substantial population of early-O stars in the mass range above $50 \mathrm{M}_{\odot}$. Interestingly, ISO observations of the [CII] $128 \mu \mathrm{m}$ line in a sample 
of ultraluminous starbursts indicate a similarly soft radiation field (Luhman et al. 1998). Could a search for WR stars reveal the same discrepancy as in the less luminous object NGC 1614 ?

The duration and age of the star-formation event. Synthesis models for WR and $\mathrm{OB}$ populations predict a rather narrow age range over which WR stars are observable. The WR phase sets in 2 to $3 \mathrm{Myr}$ after the onset of star formation and lasts for about 3 to $4 \mathrm{Myr}$ in single-star models with solar chemical composition (Meynet 1995; Schaerer \& Vacca 1998). The WR phase is extended for another $\sim 10 \mathrm{Myr}$ if additional WR stars are generated via Roche-lobe overflow in binaries (Cerviño et al. 1997; Vanbeveren et al. 1998). The observed WR/O numbers in WR galaxies exceed the ratio expected from simple WR- and Olifetime arguments ( $\sim 0.1$; Maeder \& Conti 1994). This can be understood if there is no evolutionary equilibrium because star formation is not continuous but rather peaked. Since WR stars appear and disappear abruptly (on a starformation timescale), they can serve as standard chronometers if an independent age estimate is required. Star-forming galaxies with and without Ly $\alpha$ emission are an application. Weak or absent Ly $\alpha$ in starbursts has been suggested as an age effect (Valls-Gabaud 1993), an interpretation that can be tested using starbursts with and without WR features.

\section{Single stellar populations as testbeds}

Analysis of starburst populations has the advantage of much improved number statistics. The well-studied WR galaxy NGC 1741 contains $\sim 500 \mathrm{WR}$ stars in one starburst region (Conti et al. 1996) - more than the total number of individual WR stars classified in the Local Group. The price paid for the improved statistics is the loss of spatial resolution: $100 \mathrm{pc}$ (roughly the extent of the 30 Dor cluster) subtend an angle of $1^{\prime \prime}$ at the distance of Virgo $(20 \mathrm{Mpc})$. All the details of the very early star-formation process in the 30 Dor center would be lost, and only a time-averaged star-formation history can be derived. In this section I will address the coevality of starburst populations from an extragalactic point of view. My goal is to define single (= coeval) stellar populations (SSP) and to show how SSPs permit observational tests for formation mechanisms of WR stars from single vs. binary stars and from low- vs. high-mass O stars.

In the following I will assume that the massive-star IMF is well approximated by a Salpeter power law and that this IMF is independent of galaxy parameters, in particular of metallicity. Arguments in favor of this assumption have been given by Leitherer (1998) and Massey (1998).

Some key observations were made in the nearby starburst galaxies M 82 and and M 83. Satyapal et al. (1997) found evidence for almost instantaneous, yet propagating star formation in the central 100 pc of M 82 from the anti-correlation of the CO index (indicative of red supergiants - RSG) and the $\mathrm{Br} \gamma$ emission (indicative of O stars). More quantitatively, Puxley et al. (1997) modeled the anti-correlation of $\mathrm{CO}$ and $\mathrm{Br} \gamma$ in several starburst clusters in M 83 with young populations having different age spread. An upper limit of about $5 \mathrm{Myr}$ for the age spread in individual clusters was found.

Observations at sufficiently high spatial resolution to isolate individual starburst regions show no age spread of more than $2-3 \mathrm{Myr}$ in the formation of 
massive stars. Ho \& Filippenko (1996) detected RSG features in the super star clusters in NGC 1569 and NGC 1705 but none in NGC 4214. The formation of the first RSGs occurs after about $6 \mathrm{Myr}$ according to stellar evolution models. From the absence of O-star wind features in NGC 1705, Heckman \& Leitherer (1997) find an age of at least $10 \mathrm{Myr}$, whereas the presence of such features in NGC 4214 implies an age of less than $5 \mathrm{Myr}$. The anti-correlation of O-star and RSG lines can only be understood if there is no age spread of more than 2-3 Myr.

The answer to the question 'Is the population coeval?' is 'It depends on how closely you look!'. Evidence from galaxies like M 82 and M 83 suggests that star formation is almost instantaneous in discrete cells, and then triggers subsequent events at propagation velocities of $50-100 \mathrm{~km} \mathrm{~s}^{-1}$. Considering an age spread of less than 5 Myr as coeval, starburst regions much smaller than 100 pc are almost coeval, whereas much larger regions have mixed populations. The latter case was convincingly made by Calzetti (1997) whose large-aperture IR spectroscopy of a UV selected starburst sample indicates a tight correlation between the Ostar and RSG-population over kpc scales. The correlation can be understood in terms of star-formation equilibrium due to superposition of numerous individual starbursts with different ages.

Coeval populations, and super star clusters in particular, offer unique observational tests of different WR formation channels. The traditional point of view is that most WR stars evolve from $\mathrm{O}$ stars with initial masses above $\sim 30 \mathrm{M}_{\odot}$ (for solar composition). Mass exchange in close binaries leads to the formation of additional WR stars out of $\sim 20 \mathrm{M}_{\odot}$ progenitors (D. Vanbeveren, these Proceedings). Alternatively, enhanced mass loss and/or mixing and rotation may turn single late-O stars from the same mass range into WR stars as well. Both the single-late-O and the binary mechanism for the WR formation have in common a relatively low progenitor mass. For SSPs, the low progenitor mass translates into an additional, late ( 7 to $20 \mathrm{Myr}$ after the initial star formation) WR phase. Synthesis models make very specific predictions for a starburst region observed in this late WR phase:

- A rich WR population is present when most massive stars have already evolved beyond the O-star phase. Models not taking into account binary evolution predict no $\mathrm{O}$ stars at all at $t>10 \mathrm{Myr}$. As discussed by Van Bever \& Vanbeveren (1998), mass transfer in close binaries leads to the formation of a population of blue stragglers mimicking a younger $\mathrm{O}$ population. Dense starburst clusters are particularly well-suited to test these ideas. In contrast, OB associations in the Galaxy are by far too sparse to permit statistically meaningful tests. Combining stellar evolutionary synthesis with nebular photoionization models may give additional clues. The hardness of the emission-line spectrum is critically affected by even a few hot WR stars in an aged starburst.

- WR stars and RSGs should rarely be observed simultaneously if WR progenitor masses are above $30 \mathrm{M}_{\odot}$. Both phases are too short to provide significant overlap in a SSP. WR stars and RSGs are predicted by synthesis models only if there is an age spread of several Myr during the star formation. Therefore the detection of WR features in the NGC 1569 super star cluster by GonzálezDelgado et al. (1997) came as a surprise. Taken at face value, this suggests that the massive stars in the super star cluster in NGC 1569 could not have formed instantaneously. Subsequently De Marchi et al. (1997) demonstrated that this 
cluster is double, either as a chance projection or by physical association. It is possible that RSGs and WR stars are not in the same cluster, and instantaneous bursts have occurred individually. Alternatively, the NGC 1569 star cluster may turn out as the Rosetta Stone for binary WR models.

\section{Comparison of Wolf-Rayet synthesis models}

I asked the major groups calculating synthesis models for WR populations to provide me with their predictions for a standard burst population. A comparison of their results should shed light on the internal consistency of the models and on the influence of evolutionary tracks and stellar atmospheres. A main objective is to highlight differences between single and binary WR models.

Table 2. Details of the synthesis codes used in the model comparison.

\begin{tabular}{|c|c|c|c|c|}
\hline group & authors & formation channel & evolution & atmospheres $^{a}$ \\
\hline Baltimore & Leitherer et al. & single $+0 \%$ binary & Geneva & $\begin{array}{l}\text { Lejeune et al. (1997) } \\
\text { Schmutz et al. (1992) }\end{array}$ \\
\hline Madrid & Cerviño \& Mas-Hesse & single $+30 \%$ binary & $\begin{array}{l}\text { Geneva } \\
\text { Brussels }\end{array}$ & $\begin{array}{l}\text { Kurucz (1992) } \\
\text { Mihalas (1992) }\end{array}$ \\
\hline Quebec & Dionne \& Robert & single $+50 \%$ binary & $\begin{array}{l}\text { Geneva } \\
\text { Brussels }\end{array}$ & $\begin{array}{l}\text { Kurucz (1992) } \\
\text { Schmutz et al. (1992) }\end{array}$ \\
\hline Toulouse & Schaerer \& Vacca & single $+20 \%$ binary & $\begin{array}{l}\text { Geneva } \\
\text { Brussels }\end{array}$ & $\begin{array}{l}\text { Kurucz (1992) } \\
\text { Schaerer et al. (1996) } \\
\text { Schmutz et al. (1992) }\end{array}$ \\
\hline
\end{tabular}

${ }^{a}$ Atmospheres used for WR stars are in bold face

The input physics used by the groups participating in this exercise is summarized in Table 2. More details can be found in the contributions of the listed authors in these proceedings. The four groups adopt the latest set of the Geneva tracks for single-star evolution, and an optional inclusion of WRs from binaries calculated with the Brussels code (De Loore \& Vanbeveren 1994). Baltimore and Toulouse adopt the high $\dot{M}$ Geneva tracks, whereas Madrid and Quebec use those with standard rates. The binary fraction ranges between $0 \%$ (Baltimore) to $50 \%$ (Quebec). The Toulouse models use spherically extended COSTAR-atmospheres for all pre-WR phases; the models from the three other groups assume planeparallel atmospheres. The WR phases are computed with the extended non-LTE atmospheres of Schmutz et al. (1992) in the Baltimore, Quebec, and Toulouse codes, and with plane-parallel Mihalas (1972) atmospheres in the Madrid code. The starburst parameters are: SSP with total mass $1 \mathrm{M}_{\odot}$, Salpeter IMF between 1 and $100 \mathrm{M}_{\odot}, \mathrm{Z}_{\odot}$, age range 1 to $10 \mathrm{Myr}$ in steps of $1 \mathrm{Myr}$.

The predicted WR/O ratios are plotted in Fig. 2 (left). The single-star models predict peak ratios around 0.1 , with a variation of about a factor of 3. The four groups make consistent predictions, as expected from the identical set of evolutionary tracks. Note that $\mathrm{WR} / \mathrm{O}$ is higher in the Baltimore and Toulouse models due to the adopted high $\dot{M}$. The single-WR phase ends at about $5 \pm 1 \mathrm{Myr}$ and is replaced by the binary-dominated phase between 6 and 

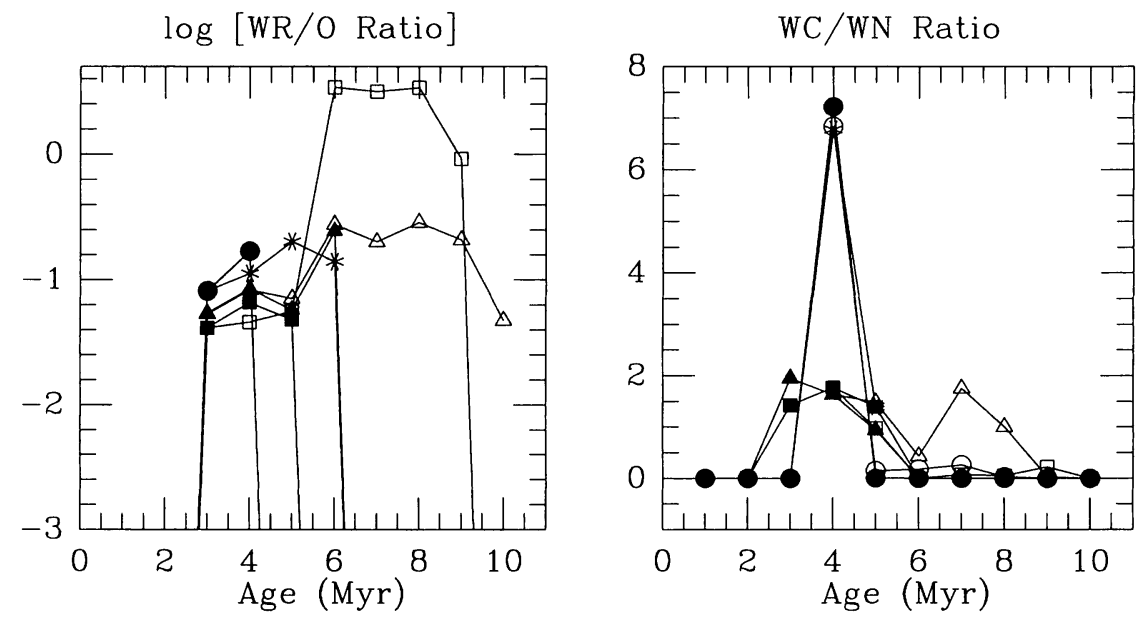

Figure 2. Predicted WR/O (left) and WC/WN (right) ratios. SSP with $Z=\mathrm{Z}_{\odot}$; Salpeter IMF normalized to total mass $1 \mathrm{M}_{\odot}$ between 1 and $100 \mathrm{M}_{\odot}$. Stars - Baltimore; filled/open squares - Madrid single/binary; filled/open triangles - Quebec single/binary; filled/open circles - Toulouse single/binary.

10 Myr. The high WR/O ratio during this epoch results from a combination of large WR- and small O-star numbers. If an independent age estimate is available for a starburst region (e.g., from RSG numbers), the predictions of Fig. 1 at later ages are useful to evaluate the importance of WR stars from low-mass progenitors. As a cautionary note, the Toulouse models assume a restrictive definition of an $\mathrm{O} \operatorname{star}\left(T_{\text {eff }}>33000 \mathrm{~K}\right)$ so that after $4 \mathrm{Myr}$ the O-star population formally becomes 0 . This serves as a warning that the predicted stellar types are not a useful quantity for comparison with observations since they are strongly dependent on the adopted definition. Quantities such as the $\lambda 4686$ (for WR stars) and $\mathrm{H} \beta$ (for $\mathrm{O}$ stars) equivalent widths should be used instead.

The right panel of Fig. 2 shows the WC/WN ratios. The most conspicuous feature is the high ratio of $\sim 7$ at $t=4 \mathrm{Myr}$ in the Baltimore and Toulouse models. This results from the high mass-loss rates which produce a large number of WC stars out of WNs. The jury is still out if this prediction is correct since few starburst regions with solar chemical compositions containing WR stars have been studied.

Next I address the mass returned to the interstellar medium by stellar winds (Fig. 3). Only results from the Baltimore, Madrid, and Quebec groups are available. The predictions for the same epoch differ by up to an order of magnitude, even within single-star models. The mass-loss rates are not always those adopted in the evolutionary tracks but from other empirical or theoretical prescriptions. This raises the concern of self-consistency. However, $\dot{M}$ in evolution models to some degree is an adjustable parameter to reproduce the gross evolutionary behavior. It may well differ from the observed mass-loss rate, given the inherent deficiencies of evolution models, such as the inadequate treatment of stellar rota- 

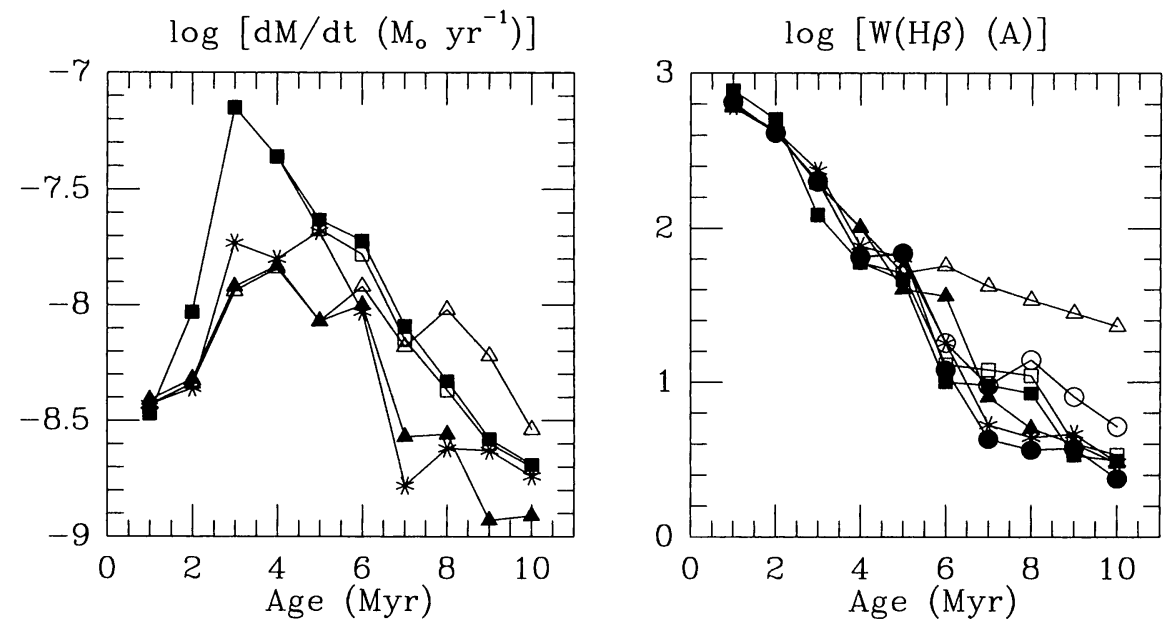

Figure 3. Mass-loss rate (left) and $\mathrm{H} \beta$ equivalent widths (right). SSP with $Z=\mathrm{Z}_{\odot} ;$ Salpeter IMF normalized to total mass $1 \mathrm{M}_{\odot}$ between 1 and $100 \mathrm{M}_{\odot}$. Stars - Baltimore; filled/open squares - Madrid single/binary; filled/open triangles - Quebec single/binary; filled/open circles - Toulouse single/binary.

tion (A. Maeder, these Proceedings). The vastly different predictions for $\dot{M}$ (and for the mechanical luminosity as well) are relevant for hydrodynamical models of starburst-driven galactic outflows, which adopt population synthesis models to compute the available energy release from stellar winds and supernovae (e.g., Suchkov et al. 1994). Supernovae would appear in Fig. 3 (left) around $5 \mathrm{Myr}$ with a constant $\dot{M} \approx 10^{-9} \mathrm{M}_{\odot} \mathrm{yr}^{-1}$. In the absence of binary WRs, supernovae become important for the mass return after about $7 \mathrm{Myr}$. If WRs from binaries are present, their mass return is a factor of several higher than that of supernovae for an additional 3 to $5 \mathrm{Myr}$.

The $\mathrm{H} \beta$ equivalent width is in the right panel of Fig. 3. The continuum does not include nebular free-free and free-bound emission in order to facilitate the model comparison. Inclusion of nebular emission will decrease the equivalent width by up to a factor of 2 during the first few Myr. It is gratifying that all models are essentially identical during the O-star dominated phase $(t<4 \mathrm{Myr})$. Plane-parallel vs. extended and LTE vs. non-LTE atmospheres do not predict significantly different $\mathrm{H}$ ionizing photon numbers. This is a conclusion reached from an extragalactic perspective: given the observational limitations (age uncertainties, photon leakage and absorption, etc.), all tested synthesis models are equally useful (or useless) for Balmer photon counting. Conversely, systematic differences of the Lyman continuum at the 0.1 dex level exist between extended and plane-parallel atmospheres (Schaerer \& de Koter 1997). Such differences are quite significant for atmospheric analysis of individual hot stars.

The model predictions do differ drastically for the stellar ionizing continuum above 4 Ryd. To illustrate this point, I compiled six models using the Baltimore and Toulouse codes (single stars only). In Fig. 4 I have plotted the number of photons above 4 Ryd predicted by different combinations of model atmospheres 


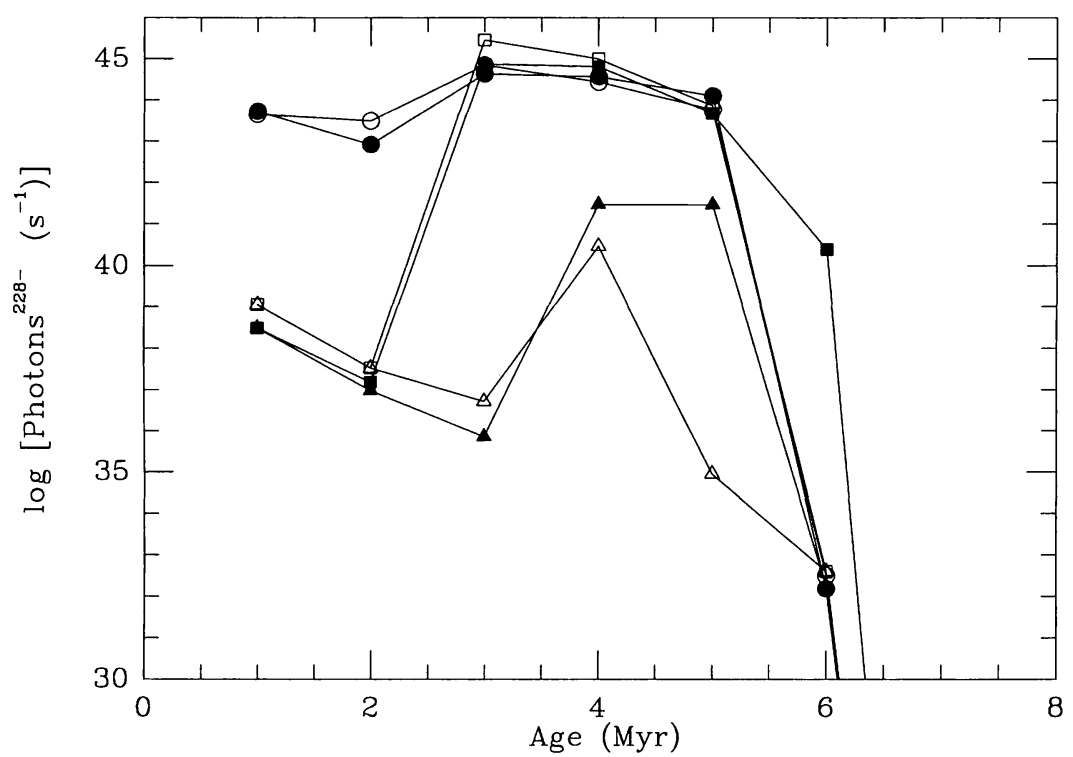

Figure 4. Number of photons with energy above 4 Ryd. SSP with $Z=\mathrm{Z}_{\odot}$; Salpeter IMF normalized to total mass $1 \mathrm{M}_{\odot}$ between 1 and $100 \mathrm{M}_{\odot}$; no binaries. Triangles - Kurucz for O and WR stars; squares - Kurucz for O stars, Schmutz for WR stars; circles - Schaerer for O stars, Schmutz for WR stars; filled symbols - Geneva high $\dot{M}$; open symbols - Geneva standard $\dot{M}$.

and evolutionary tracks. The combinations are listed in the figure caption. At epochs earlier than $3 \mathrm{Myr}$, O stars provide the photon flux. The mass-loss prescription is irrelevant. The extended COSTAR-atmospheres (Schaerer et al. 1996) predict photon fluxes which are five orders of magnitude above those predicted by plane-parallel Kurucz (1992) atmospheres. The observational significance of the predicted photon flux during the early starburst phase is probably minor: even with Costar atmospheres the number of photons with $\lambda<228 \AA$ is still two to three orders of magnitude below those with $\lambda<912 \AA$ so that He II emission will be hard to detect.

The WR phase begins and ends around 3 and $6 \mathrm{Myr}$, respectively. The extended WR atmospheres of Schmutz et al. (1992) predict up to 10 orders of magnitude higher fluxes than the plane-parallel Kurucz atmospheres. The details of the flux enhancement depend on the specific wind densities which can either increase the EUV fluxes by desaturating the $\mathrm{He}^{+}$ground level or decrease them by density induced recombination of helium in the wind. The photon fluxes are two orders of magnitude higher than in the pre-WR phase. Significant nebular He II recombination is predicted by these models during the WR phase (D.Schaerer, these Proceedings). The different mass-loss prescriptions become noticeable during the end of the WR phase. Models with high mass loss produce hotter WR stars in larger numbers so that even plane-parallel Kurucz atmospheres have a relatively high output of photons above 4 Ryd. 


\section{Current state of Wolf-Rayet synthesis and future directions}

Populations of WR stars have been detected in galaxies as distant as $\sim 0.5 \mathrm{Gpc}$ (Armus et al. 1988). Could the broad emission feature at $1486 \AA$ in the $z=2.72$ galaxy 1512-cB58 (Pettini et al. 1997) be due to WN stars? Quantitative analysis of spectral signatures from WR populations rests on population synthesis models. Such models are becoming increasingly refined owing to progress in stellar atmosphere and evolution models.

The purpose of this review was to highlight applications of synthesis models and to perform a critical evaluation of their reliability. Not unexpectedly, synthesis models are subject to the same uncertainties as WR atmospheres and evolution models. Major issues are:

- The formation channel for WR stars. There is overwhelming evidence that the most massive $\mathrm{O}$ stars lose sufficient mass via radiatively driven winds and occasional instabilities that they evolve into WR stars. This evolutionary path was originally suggested by Conti (1976). What about less massive stars? Stars in the $20 \mathrm{M}_{\odot}$ range could turn into WRs either via Roche-lobe overflow in binaries and/or strong mass loss during the RSG phase. Massive, coeval starburst clusters allow specific observational tests to support or reject one or the other evolution scenario using synthesis models. Such tests are difficult, if not impossible to perform in sparse Galactic OB associations.

- The ionizing continuum of WR stars. The EUV spectral region above 4 Ryd of a young starburst population is almost always dominated by WR radiation. Despite impressive progress over the past 10 years, the field of expanding WR atmospheres is still rather experimental. A major unresolved issue is the treatment of line-blanketing in expanding atmospheres and its dependence on metallicity. Note that all WR atmospheres used for the model comparison are unblanketed (see Table 2). Future emphasis should be on utilizing photoionization models for starbursts to probe the EUV spectrum of a WR population. This is another example where observations of integrated WR spectra may provide an advantage over single-star data. G. Stasińska discusses such an approach in her review.

- The definition of WR stars. Are we sure that objects called WR stars in synthesis models are identical to those classified spectroscopically? Synthesis models rely mostly on the evolutionary definition of a WR star, i.e. its central and surface helium content. In most cases the connection between this and the spectroscopic definition has been verified but notable exceptions exist: a massive star with strong mass loss may mimic a WR spectrum although it may very well be rather unevolved in the evolutionary sense. S. Heap (these Proceedings) reports such cases. How common are these wolves in sheep's clothing?

The theme of this conference was the interplay between the stellar, individual and the extragalactic, integrated properties of WR stars. Synthesis models for any population species will always benefit from progress in both directions. In the case of WR populations, comparison between observations and synthesis models will currently tell us less about the star-formation process and starburst evolution than about WR astrophysics. Given the rapid progress in our understanding of $W R$ stars over the past years, we may hope that WR stars will become standard candles by the time of the next Wolf-Rayet symposium. 
Acknowledgments. I am grateful to Miguel Cerviño, Danny Dionne, Miguel Mas-Hesse, and Daniel Schaerer for providing their model calculations. Careful reading and helpful comments by Daniel Schaerer and Grażina Stasińska are appreciated.

\section{References}

Armus, L. Heckman, T.M., Miley, G.K. 1988, ApJ 326, L45

Calzetti, D. 1997, AJ 113, 162

Cerviño, M., Mas-Hesse, J.M., Kunth, D. 1997 in: J. Franco, R. Terlevich \& A. Serrano (eds.) Starburst Activity in Galaxies, RevMexAA Ser. Conf. 6, 188

Cid Fernandes, R., Terlevich, R. 1995, MNRAS 272, 423

Conti, P.S. 1976, Mém. Soc. Roy. Sci. Liège, 6e Sér., Tome IX, 193

- , 1991, ApJ 377, 115

Conti, P.S., Leitherer, C., Vacca, W.D. 1996, ApJ 461, L87

de Koter, A., Heap, S.R., Hubeny, I. 1997, ApJ 477, 792

De Loore, C., Vanbeveren, D. 1994, A\&AS 105, 21

De Marchi, G., Clampin, M., Greggio, L., Leitherer, C., Nota, A., Tosi, M. 1997, ApJ 479, L27

Doyon, R., Puxley, P.J., Joseph, R.D. 1992, ApJ 397, 117

Gabler, R., Gabler, A., Kudritzki, R.P., Puls, J., Pauldrach, A. 1989, A\&A 226, 162

Goldader, J.D., Joseph, R.D., Doyon, R., Sanders, D.B. 1997, ApJ 474, 104

González-Delgado, R.M., Heckman, T.M., Leitherer, C., Meurer, G., Krolik, J., Wilson, A.S., Kinney, A.L., Koratkar, A. 1998, ApJ 505, 174

González-Delgado, R.M., Leitherer, C., Heckman, T.M., Cerviño, M. 1997, ApJ 483, 705

Heckman, T.M., González-Delgado, R.M., Leitherer, C., Meurer, G.R., Krolik, J., Wilson, A.S., Koratkar, A., Kinney, A. 1997, ApJ 482, 114

Heckman, T.M., Leitherer, C. 1997, AJ 114, 69

Ho, L.C., Filippenko, A.V. 1996, ApJ 472, 600

Kurucz, R.L. 1992, in: B. Barbuy \& A. Renzini (eds.), The Stellar Populations of Galaxies, Proc. IAU Symp. No. 149 (Dordrecht: Kluwer), p. 225

Lançon, A., Rocca-Volmerange, B. 1996, New Astron. 1, 215

Leitherer, C. 1998, in: G. Gilmore \& D. Howell (eds.), The Stellar Initial Mass Function, Proc. 38th Herstmonceux Conference, ASP-CS 142, 61

Leitherer, C., Schaerer, D., Goldader, J.D., González-Delgado, R., Robert, C., Foo Kune, D., de Mello, D.F., Devost, D., Heckman, T.M. 1999, ApJS submitted

Lejeune, T., Buser, R., Cuisinier, F. 1997, A\&AS 125, 229

Luhman, M.L., Satyapal, S., Fischer, J., Wolfire, M.G., Cox, P., Lord, S.D., Smith, H.A., Stacey, G.J., Unger, S.J. 1998, ApJ 504, L11

Maeder A., Conti, P.S. 1994, ARAA 32, 227

Massey, P. 1998, in: G. Gilmore \& D. Howell (eds.), The Stellar Initial Mass Function, Proc. 38th Herstmonceux Conference, ASP-CS 142, 17

Meynet, G. 1995, A\&A 298, 767

Mihalas, D. 1972, Non-LTE Model Atmospheres for B and O Stars, NCAR-TN/STR-76, (Boulder: NCAR)

Parker, J.W., Heap, S.R., Malumuth, E.M. 1995, ApJ 448, 705 
Perry, J.J., \& Dyson, J.E. 1985, MNRAS 213, 665

Pettini, M., Steidel, C.C., Dickinson, M., Kellogg, M., Giavalisco, M., Adelberger, K.L. 1997, in: W.H. Waller, M.N. Fanelli, J.E. Hollis \& A.C. Danks (eds.) The Ultraviolet Universe at Low and High Redshift (Woodbury: AIP), p. 279

Puxley, P.J., Doyon, R., Ward, M.J. 1997, ApJ 476, 120

Satyapal, S., Watson, D.M., Pipher, J.L., Forrest, W.J., Greenhouse, M.A., Smith, H.A., Fischer, J., Woodward, C.E. 1997, ApJ 483, 148

Schaerer, D., Contini, T., Kunth, D. 1999, A\&A 341, 399

Schaerer, D., de Koter, A. 1997, A\&A 322, 598

Schaerer, D., de Koter, A., Schmutz, W., Maeder, A. 1996, A\&A 310, 837

Schaerer, D., Vacca, W.D. 1998, ApJ 497, 618

Schmutz, W., Leitherer, C., Gruenwald, R. 1992, PASP 104, 1164

Storchi-Bergmann, T., Cid Fernandes, R., Schmitt, H.R. 1998, ApJ 501, 94

Suchkov, A.A., Balsara, D.S., Heckman, T.M., Leitherer, C. 1994, ApJ 430, 511

Terlevich, R., Melnick, J. 1985, MNRAS 213, 841

Vacca, W.D., Robert, C., Leitherer, C., Conti, P.S. 1995, ApJ 444, 647

Valls-Gabaud, D. 1993, ApJ 419, 7

Van Bever, J., Vanbeveren, D. 1998, A\&A 334, 21

Vanbeveren, D., Van Rensbergen, W., de Loore, C. 1998, The Brightest Binaries (Dordrecht: Kluwer)

\section{Discussion}

Shara: (1) Could you use this methodology to directly measure, or strongly constrain the WR star binary fraction in a sample of galaxies? (2) What would be the most extreme (and observationally detectable) effect for a very high binary fraction, say $80 \%$ (as in the SMC)?

Leitherer: (1) I would presume the most promising method is to search for the simultaneous presence of RSGs and WR stars. They should not be found at the same time in large numbers for instantaneous bursts forming WR stars only from single stars. (2) The question of course is if all the binaries are really interacting. I doubt that this is actually the case for all SMC objects. Assuming that they are, the Brussels models (Vanbeveren and coworkers, these Proceedings) make very specific predictions for different binary fractions.

Crowther: New WR atmospheric models including line-blanketing plus a metallicity dependence of $\dot{M}$ predict a dramatically harder $\lambda<228 \AA$ flux-distribution at low metallicity. So my question is, do we observe a higher proportion of WR galaxies at low $Z$ showing nebular He II 4686 emission?

Leitherer: There is indeed a trend for stronger stellar and nebular He II 4686 for lower metallicity. However, I don't think selection effects have been properly addressed yet. Searches for WR galaxies tend to be biased against finding metal-rich objects, which tend to be larger galaxies with stronger older populations. WR features (both stellar and nebular) would be harder to detect in these objects.

Koenigsberger: Could you comment on what the effect would be if you include the very massive objects Sally Heap presented and which have WR-like spectra (in R 136)?

Leitherer: The WR phase would be predicted earlier by models which include the WRlike objects in R 136. We are again back to the question of the definition of a WR star. In principle this is easy to change in the evolutionary models. The question is, what 
have we learned? The situation may not be as bad as it appears. R 136 is definitely younger than many starbursts we observe. This is indicated by its weak Si IV 1400 in the integrated spectrum, as opposed to P-Cygni profiles often observed in starbursts. Starbursts are often older and the 'pseudo-WRs' may have already disappeared.

Terlevich: I have to insist that I'm worried about the factor of two difference between the observed $W_{\mathrm{H} \beta}$ and the predicted ones. We should know to which of the many possible effects is this difference due. I find it interesting that line ratios seem to be better predicted by models than equivalent widths.

Leitherer: I fully agree that there is a discrepancy. It may indicate that the real galaxy is more complicated than our simple models. Dilution by un underlying population and/or photon leakage could systematically lower the equivalent widths. Another possibility is obscuration of the very early starburst by gas and dust! This is addressed in a two poster papers by Beck and Turner (these Proceedings).

Vanbeveren: As I said during my talk, the effect of accretion stars (mass gainers) is essential and this evolution after mass transfer (and after the SN explosion of the primary) must be followed in detail. This is not always done by those who include binaries in their population-synthesis models.

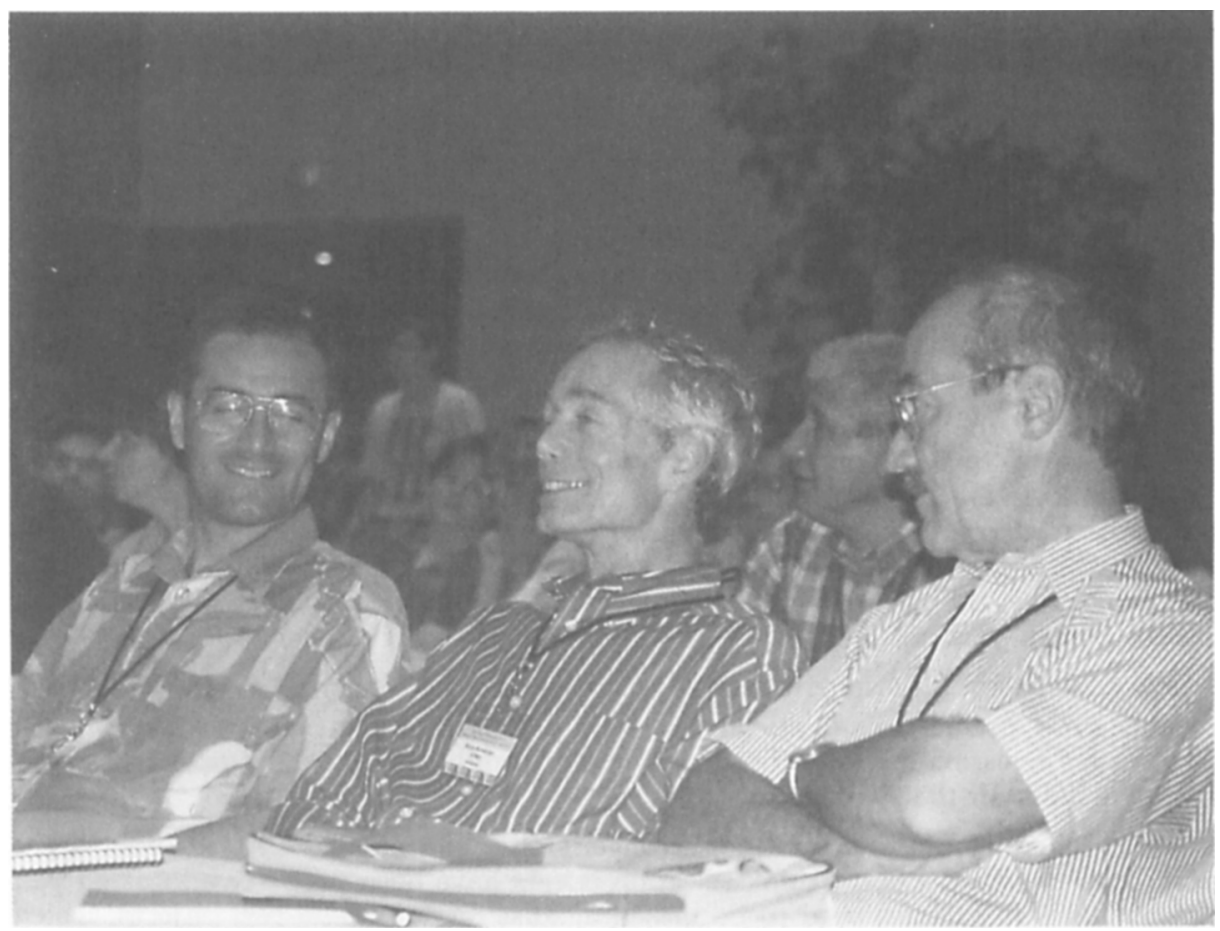

